\title{
Workshop on Object Recognition for Input and Mobile Interaction
}

Hui-Shyong Yeo

School Of Computer Science

University of St Andrews, UK

hsy@st-andrews.ac.uk

\section{Gierad Laput}

School of Computer Science

Carnegie Mellon University

gierad.laput@cs.cmu.edu
Nicholas Gillian

Google, ATAP

Mountain view, CA, USA

ngillian@google.com

\section{Aaron Quigley}

School Of Computer Science

University of St Andrews, UK

aquigley@st-andrews.ac.uk

Paste the appropriate copyright statement here. ACM now supports three different copyright statements:

ACM copyright: ACM holds the copyright on the work. This is the historical approach.

License: The author(s) retain copyright, but ACM receives an exclusive

publication license.

Open Access: The author(s) wish to pay for the work to be open access. The additional fee must be paid to ACM.

This text field is large enough to hold the appropriate release statement assuming it is single spaced in a sans-serif 7 point font.

Every submission will be assigned their own unique DOI string to be included here.

\begin{abstract}
Today we can see an increasing number of Object Recognition systems of very different sizes, portability, embedability and form factors which are starting to become part of the ubiquitous, tangible, mobile and wearable computing ecosystems that we might make use of in our daily lives. These systems rely on a variety of technologies including computer vision, radar, acoustic sensing, tagging and smart objects.
\end{abstract}

Such systems open up a wide-range of new forms of touchless interaction. With systems deployed in mobile products then using everyday objects that can be found in the office or home, we can realise new applications and novel types of interaction. Object based interactions might revolutionise how people interact with a computer. System could be used in conjunction with a mobile phone, for example it could be trained to open a recipe app when you hold a phone to your stomach, or change its settings when operating with a gloved hand.

Although the last few years have seen an increasing amount of research in this area, knowledge about this subject remains under explored, fragmented, and cuts across a set of related but heterogeneous issues. This workshop brings together researchers and practitioners interested in the challenges posed by Object Recognition for Input and Mobile 


\section{Interaction.}

\section{ACM Classification Keywords}

H.5.2 [Information Interfaces and Presentation(e.g., $\mathrm{HCl}$ )]: User Interfaces-Evaluation/methodology, Input devices and strategies, Interaction styles; B.4.2 [Input/Output and Data Communications]: Interconnections (Subsystems)Interfaces; D.2.2 [Software Engineering]: Design Tools and Techniques-User Interfaces

\section{Author Keywords}

Radar; Context-Aware Interaction; Material Classification; Object Recognition; Ubiquitous Computing; Interaction Techniques and Mobile Computing

\section{Introduction}

The objective of this workshop is to bring together researchers active and interested in the areas of object recognition for input for mobile interaction to share approaches and experiences, identify research and deployment challenges, and envision the next generation of applications that rely on widely deployed sensor systems in not one but an ecosystem of touchless interaction devices. Consider the example of sensors being embedded in a mobile and wristworn device, how might the relative orientation of movement of objects between those two points drive interaction. While there are a range of issues to consider, this workshop focusses on the two significant objectives of infrastructures and objects as sites for interaction.

There are many challenges with building the underlying system infrastructures for object recognition for mobile interaction. For example, what are the standards and operating system requirements? How can vision, radar [2] or acoustic sensing [1] or tagging systems which exist in multiple mobile and wearable elements act in a coordinated man- ner to reliably determine object interaction? What types of software libraries, simulators and IDEs are required for development? How can we ensure inter-device interoperability in the face of heterogeneous device configurations and varying approaches to object recognition?

In addition, there are significant challenges in the design and deployment of these kinds of object based interfaces. For example, what are the implications of interfaces that rely on sensing the movement and type of passive, physical objects? How might multiple points of sensing on, and around the body open up new design considerations? Which elements of the interface are best distributed in outputs? What new interaction techniques are necessary in these environments? What are the performance, comfort, and preference consequences of relying of object-interaction as a mobile interface?

This workshop will discuss computer vision and miniature radar systems which are being developed to sense micro and subtle motion of human fingers being repurposed to recognise objects. One such system is the Project Soli by the Google ATAP (Advanced Technology and Projects). Participants are encouraged to bring other mobile and desktop based systems suitable for object recognition for mobile interaction.

\section{Topics of Interest}

The following eight topics of interest will be specified in our general call for papers for this workshop. These topics include:

- Understanding the design space and identifying factors that influence user interactions in this space

- Developing evaluation strategies to cope with the complex nature of computer vision or radar-based 


\section{object interaction}

- Ethnography and user studies of tangible and object interaction

- Examples of applications of object interaction

- Social factors that influence the design of suitable interaction techniques for object interaction

- Exploring interaction techniques that facilitate multiperson object interaction

- Novel input mechanisms for single and multi-point sensing systems (eg. in mobile, tablet and wrist worn device)

- SDK/APIs, IDEs, and hardware platforms for the development of object recognition

\section{Audience}

We invite contributions from researchers and practitioners working in the area of object recognition and interaction. Our goal is to develop an audience who could share approaches and experiences, identify research and deployment challenges, and envision the next generation of applications that rely on object recognition and interaction. Such systems can be embedded in personal, wearable and mobile devices i.e. physically decoupled in different ways yet are virtually coupled due to the interactions they support. Researchers who attend the workshop are working on different views of the problem (e.g., at the interaction technique, application, middleware or hardware level), with a wide range of sensing systems and interaction technologies (e.g., Project Soli, computer vision, alternative hardware, ...), and in a wide range of object interaction applications (e.g., education, on-the-go interaction, medicine, smart sensing). Such interface are nacent yet we are on the cusp of such interfaces becoming mainstream and this workshop allows this community to develop a wider research agenda.

\section{Workshop}

The workshop lasts a full day and is structured to provide maximum time for group discussion and brainstorming Each participant is familiar with all position papers (which were made available to them well in advance of the event). The workshop is structured around four sessions (separated by the morning break, lunch and afternoon break). In the first session the participants briefly introduce themselves and engage in a brainstorm session to outline key discussion topics for the two midday sessions. In the second and third sessions the group is divided into sub-groups moderated by the workshop organisers to have focused discussions on some of the key topics identified earlier. In the fourth session the group reconvenes to both summarise the advances identified in the breakout discussions and to identify next steps. Next steps may include plans for a COST action submission, special issue of a Journal or book and planned research collaborations.

\section{Workshop Organisers}

Hui-Shyong Yeo is a $\mathrm{PhD}$ student in $\mathrm{SACHI}$ in the Schoo of Computer Science at the University of St Andrews. Before that he worked as a researcher in KAIST for one year. Yeo has a wide range of interest within the field of $\mathrm{HCl}$, including topics such as wearable, gestures, mixed reality and text entry. Currently he is focusing on single-handed interaction for his dissertation topic. He has published in conferences such as CHI '16 and CHI '17 UIST '16, MobileHCl '16 (honourable mention), SIGGRAPH and journals such as MTAP and JNCA.

Gierad Laput is a Ph.D. student at the School of Computer Science at Carnegie Mellon University, where he specializes in Human-Computer Interaction. His research focuses 
on building and studying ubiquitous sensing technologies for mobile devices, wearable computing, and smart environments. His work has received multiple best paper awards and best paper nominations at premier venues in human-computer interaction. His work has been recognized by Fast Company as one of the 10 Innovation by Design Awards recipients in 2016, and he has received an Adobe Research Fellowship in Human-Computer Interaction, and a Qualcomm Innovation Fellowship. He holds a bachelor's degree in Electrical Engineering, and a master's degree in Human-Computer Interaction from the University of Michigan.

Nicholas Gillian is an engineer and creative technologist specializing in gesture recognition, machine learning and software architecture in the Google's Advanced Technology and Projects group, where he leads the machine learning for Project Soli. With a background in Gesture Recognition for Musician Computer Interaction from the Sonic Arts Research Center in Queen's University Belfast, he has previously worked for Samsung, a machine-learning consulting company and as a post-doctoral associate and Fulbright Scholar at the MIT Media Lab, with the Responsive Environments research group.

Aaron Quigley is the Chair of Human Computer Interaction and deputy head of school in the School of Computer Science at the University of St Andrews, UK. Aaron's research interests include surface and multi-display computing, human computer interaction, pervasive and ubiquitous computing and information visualisation. He has published over 135 internationally peer-reviewed publications including edited volumes, journal papers, book chapters, conference and workshop papers and holds 3 patents. In addition he has served on over 80 program committees and has been involved in chairing roles of over 20 international conferences and workshops including UIST, ITS, CHI, Pervasive, UbiComp, Tabletop, LoCA, UM, I- $\mathrm{HCl}, \mathrm{BCS} \mathrm{HCl}$ and Mo-
bileHCl. He has organised previous conference workshops at CSCW 2016, CHI 2016, AVI 2012/10/08. He is also the ACM SIGCHI Vice President for Conferences and a convenor of the ACM Future of Computing Academy.

\section{Program Committee}

Our international program committee of experts in the field, draws on strengths from interaction design, mobile computing, user interface design, hardware design, mobile devices, human computer interaction and ethnography. The program committee currently includes:

- Andrea Bianchi, KAIST, Korea

- Nicholas Gillian, ATAP Google, USA

- Chris Harrison, Carnegie Mellon University, USA

- Niels Henze, University of Stuttgart, Germany

- Gierad Laput, Carnegie Mellon University, USA

- Rong-Hao Liang, Eindhoven University of Technology, Netherlands

- Aaron Quigley, University of St Andrews, UK

- Kaisa Väänänen, Tampere University of Technology, Finland

- Hui-Shyong Yeo, University of St Andrews, UK

\section{Acknowledgments}

The authors thank the Google Soli team for providing the hardware and software support through their Google ATAP (Advanced Technology and Projects) Project Soli alpha developer kit. 


\section{REFERENCES}

1. G. Laput, R. Xiao, and C. Harrison. Viband: High-fidelity bio-acoustic sensing using commodity smartwatch accelerometers. In Proceedings of the 29th Annual Symposium on User Interface Software and Technology, UIST '16, pages 321-333, New York, NY, USA, 2016. ACM.

2. H.-S. Yeo, G. Flamich, P. Schrempf, D. Harris-Birtill, and A. Quigley. Radarcat: Radar categorization for input \& interaction. In Proceedings of the 29th Annual Symposium on User Interface Software and Technology, UIST '16, pages 833-841, New York, NY, USA, 2016. ACM. 